\title{
Caregivers' Perspectives on Privacy in Aged Care Monitoring Devices
}

\author{
Sami ALKHATIB ${ }^{\text {a,b }}$, Jenny WAYCOTT ${ }^{\text {a }}$, George BUCHANAN a, \\ Marthie GROBLER ${ }^{\mathrm{b}}$ and Shuo WANG ${ }^{\mathrm{b}}$ \\ ${ }^{a}$ School of Computing and Information Systems, University of Melbourne, Australia \\ ${ }^{b}$ CSIRO's Data61, Melbourne, Australia
}

\begin{abstract}
As people move into advanced old age, they may experience cognitive impairments and frailty, making it difficult for them to live without support from others. Caregivers might decide to use aged care monitoring devices (ACMDs) to support older adults under their care. However, these devices raise privacy concerns as they collect and share sensitive data from the older adult's private life in order to provide monitoring capabilities. This study involved interviewing formal and informal caregivers who used/may use ACMDs to investigate their views on privacy. The study found that although caregivers consider protecting older adults' privacy important, they may overlook privacy in order to gain benefits from ACMDs. We argue that ACMD developers should simplify privacy terms and conditions so that caregivers can make well-informed decisions when deciding to use the device. They also should consider providing users with flexible privacy settings so that users can decide what data to collect, whom to share it with and when.
\end{abstract}

Keywords. aged care, caregiving, privacy, monitoring

\section{Introduction}

As people move into advanced old age, they may experience cognitive impairments and frailty, making it difficult for them to live without receiving support from others. Informal caregivers (e.g. family members) and/or formal caregivers (e.g. paid aged care workers) may be required to check on and assist older adults under their care. This can be challenging and a source of considerable distress for caregivers, in particular when they support older adults who require frequent attention [5].

Aged Care Monitoring Devices (ACMDs) support older adults living alone at home or in residential care facilities. These devices collect older adults' health and wellbeing information and share it with caregivers using the Internet. ACMDs provide caregivers with peace of mind by ensuring they will be informed whenever older adults need help [12]. Despite the expected benefits from using ACMDs, these devices pose privacy challenges as they collect and share older adults' personal details with others [1]. Caregivers using ACMDs may be able to know details from older adults' lives that might be deemed as sensitive. Moreover, ACMD service providers may use the collected data to create behavioural and health records in order to use them for unintended purposes (e.g. marketing and research) without older adults' consent.

Although caregivers might decide to use monitoring devices to help them provide better support to older adults [16], previous studies found that older adults may reject using these devices due to their privacy concerns [2]. However, only a few studies have 
investigated caregivers' perspectives in the use of ACMDs and whether older adults' privacy is one of their concerns [18]. This paper addresses this gap by exploring caregivers' privacy perception in the use of ACMDs. The findings will inform ACMD developers about caregivers' privacy concerns to help them create monitoring devices that support older adults' and retain their privacy at the same time.

\section{Study Aims}

The role of the caregivers in deciding to use ACMDs, particularly for older adults experiencing cognitive impairment, is crucial [10], [14]. However, there is limited understanding of how caregivers view of privacy in the use of ACMDs. This study aims to fill this knowledge gap by conducting in-depth interviews with informal and formal caregivers, to gain insights into how caregivers perceive privacy in the use of ACMDs and their privacy concerns that need to be addressed.

\section{Method}

We conducted an interview study with 12 caregivers ( 8 informal and 4 formal caregivers) to gain insights into how caregivers perceive privacy in ACMDs. Although 12 is a small sample size, this study aimed to gain in-depth insights rather than broad generalisations. Further, the sample size aligns with other similar studies. For instance, Caine [4] and Marshall et al. [12] found in their review studies that a considerable number of computing science empirical studies rely on around 12 participants to provide findings. To recruit participants, we contacted aged care facilities, home care service providers and ACMD companies to nominate informal/ formal caregivers as potential interviewees. We then contacted the nominated caregivers via email to inform them about the study and schedule an interview if they were interested.

Out of the 12 participants, 3 informal and 4 formal caregivers were using ACMDs, 3 informal caregivers had used ACMDs previously (e.g. GPS trackers and fall detectors). Two informal caregivers never used an ACMD. In this paper, all participants are anonymised and referred to by IP1, 2, 3 etc. for informal caregivers and FP1, 2, 3 etc. for formal caregivers.

Interviews were semi-structured; we used a set of questions as a guide, but sometimes we asked other questions to further explore interesting answers provided by the participants. The questions were open-ended so that participants could provide as much detail as they wanted to. Questions included: "what type of information do you prefer to be collected by using a monitoring device?" and "what do you know about privacy policies associated with these devices?". In addition, we read a scenario [8] based on the "Uninvited Guests" video by Superflux [17], which depicts Thomas, a man in his 70 s who received ACMDs from his children to monitor diet, activities and sleep. The scenario was used to provoke discussion about privacy and to better understand what sort of information participants felt comfortable collecting through ACMDs, when and with whom they prefer to share this information.

The interpretive approach was implemented to make sense of the interview transcripts. This involves gaining insights about the phenomenon explored by interpreting the meanings people assign to it [19]. Following an interpretive approach 
corresponds with the aim of our study of getting a better understanding of caregivers' views on privacy in the use of ACMDs.

\section{Findings}

In this section, we discuss privacy perceptions and views in the use of ACMDs that caregivers in this study had: 1) benefits versus risks, 2) limited data sharing and 3) using ACMDs, the decision is older adults' too.

\subsection{Benefits Versus Risks}

Participants indicated that using ACMDs should be well-justified whether they are used to monitor older adults living in their home or in an aged care facility. For instance, IP1 said "There has to be [for using ACMDs] a very clear justification or a rationale for it".

In order to justify using ACMDs, participants emphasised that the benefits of using these devices should outweigh any privacy risks associated with them. However, we noted that different participants had different views on what they perceived as a benefit that outweighs privacy risks. For instance, IP5 suggested that any type of monitoring is accepted as long as it extends the ability of older adults to remain in their home as they age: "That's a lesser of the evils [losing privacy] of putting mum monitored. It's my job to make sure that we respect it [her privacy] as much as practically possible. So, it's for filling her ambition in staying home and be supported". IP7, however, emphasised that although using ACMDs may enable older adults to live independently in their home, uncontrolled monitoring - by knowing personal details about older adults under their care without any restrictions - is unacceptable and likened it to surveillance. For instance, IP7 commented on Thomas's scenario and said: "I'm extremely uncomfortable with what you've just said. It's George Orwell".

Other participants drew links between the expected benefits of using ACMDs and the type of data that these devices will collect and share. They pointed out that ACMDs should only monitor health and wellbeing information required to mitigate negative consequences related to specific concerns. This should be decided based on the current needs of older adults and any known previous health or wellbeing indicators related to previously identified issues. As an example: "The one device I did use was a tracker, so that I could tell where he was. He did wander a period of time, so I did use that on several occasions" (IP4). One type of information that most of the participants agreed will be beneficial to monitor was detecting falls. Falls are common among older people and can have severe consequences. FP2 said: "Smart mat, if they [aged care residents] are at high risk of falls, that will alert us when they're trying to come out of the bed".

Similarly, formal caregivers noted that ACMDs can assist them in their work, particularly to monitor older adults who are experiencing cognitive problems. This helps caregivers ensure that older adults get the necessary treatment and remain safe. For instance, FP2 said: "Well, the wandering alarm, we really needed it because we have residents that are at high risk of absconding. We had already few situations where they actually got away and absconded". FP4 provided another example of a potential use to help provide the right treatment and said: "They've put her [resident] on a Coloxyl [treat constipation] morning and night. She might go to the toilet, but she doesn't remember and tells them that she hasn't been and so then they give her something [Coloxyl]'. 
Participants indicated that they will accept using ACMD if they perceive the benefits to outweigh privacy risks. Describing this relation, IP5 said, "Quite frankly, it's a risk benefit equation. So, what's the benefit I'm getting from doing this? And what's the risk associated with it? If I'm not going to have any risk, I'm not going to get any benefit".

\subsection{Limited Data Sharing}

Participants emphasised that ACMDs should only share information whenever these devices detect concerning indicators that require immediate intervention (e.g. falls). Otherwise, sharing a limited number of wellbeing status reports should be sufficient as long as these reports confirm that older adults are doing fine. For instance, IP5 said: "Emergencies! You don't want to know day-to-day activities. One message per day is good, it's another way of monitoring how she 's [mother living alone] going”.

Although formal caregivers prefer to only receive alarms for emergency situations, they noted that it is their responsibility to check data collected by ACMDs regularly. Formal caregivers indicated that they are guided by their workplace processes. For instance, FP2 noted that she has to regularly check a monitor to see a visual of reports from monitoring devices from residents' rooms: "Probably ... we do it every two hours".

Most participants indicated that the collected information should only be shared with certain people. IP1 said: "It's only to significant people, relevant people and only the relevant information. I would say it's the person who is in charge of care". Participants were worried about sharing collected information with unintended parties as this may lead to unintended uses such as for marketing campaigns. IP4 said: "That does worry me that somebody there knows what you're thinking, and tracking what you're looking at, and then applying marketing, and advertising, not appropriately" IP5 noted that it is unacceptable to share older adults' collected data with any third-parties. IP5 justified this by indicating that users pay money to buy a monitoring device and are not providing data in exchange for a free service, as is the case with Facebook. As a solution, IP5 suggested that companies should consider optional privacy settings; customers need to be able to determine with whom to share their data and for what purposes. For instance, ACMD users might want their data to be used for other purposes in exchange for reduced costs: "So they say, 'we'll harvest your information and sell it, I will give you a reduction in your price of the unit or here's the price of the unit and it's completely secure and private your choice'.".

\subsection{Using ACMDs, the Decision is Older Adults' Too}

Most of the participants emphasised the importance of older adults' consent when using ACMD. They believe that older adults should be aware that they are monitored by these devices and provide well-informed consent before using them. This means not to impose using ACMDs on older adults and to discuss details with them about the purpose of the device before using it. For instance, IP2 commented on Thomas's scenario and said: "If he is happy to have his daughter know those things [monitoring him], then that's fine. But if she is imposing that on dad without his consent or his agreement maybe not... And it needs to be an open consent, not a grudging one".

Although formal caregivers indicated that they discuss the purpose of new ACMD with their residents, they pointed out that residents of aged care facilities may not always be able to provide consent. FP2 emphasised that caregivers are obliged to protect their residents by taking any necessary action(s) based on their specific needs: "They have no 
choice sometimes, so they have to accept it. We do explain to them why they are having the mat [to detect if residents get out of bed]. Some residents are a bit resistive to it. They'd be like, it's my right if I want to get up and walk, I can do it'”.

However, two informal caregivers who have older relatives in residential care noted that these facilities should not take older adults' consent for granted whenever they use ACMDs to monitor them. They indicated that there are residents in aged care facilities who are not capable of making decisions by themselves and may need assistance to decide. IP1 said, "Because they can't give informed consent, it shouldn't be assumed that it's okay. It's not okay if a person can't give a consent". IP1 therefore emphasised that proxies - who are responsible for answering questions on older adults' behalf, particularly regarding health and care services [3] - should be consulted before using ACMDs, "I would object strongly to any device being used without discussions with me and without my approval, because I speak for her [wife in residential care]". Both participants indicated that privacy decisions should be respectful. Caregivers need to be well-informed about any ACMD to be used and carefully consider what is proper in terms of privacy based on older adults' circumstances. IP6 said: "I think privacy comes under to me, it would be, how would you wish to be treated if you were receiving care".

To be well-informed about what happens with the data collected by ACMDs, it is necessary to read privacy policies provided with these devices. Although privacy policies provide users with details on how service providers will handle, protect and use users' data [6], we found that only two participants consider(ed) reading privacy policies and terms and conditions before using these devices. IP8 said: "I'm the sort of person who reads all 69 pages of a document that might handle something because I want to know whether it was relevant". Other participants indicated that they simply choose to accept terms and conditions without reading them. IP5 said, "You know what!? You just flagged something that I completely ignored. We would tick the box where you just agree to terms and conditions that we haven't even read".

Participants reported two main reasons for not reading ACMD privacy policies: 1) privacy policies are too long and use technical jargon that is difficult to understand 2) some caregivers may need to use a monitoring device regardless of its privacy risks. IP8 said: "I think it's related to 2 things; the privacy policies are too long and too complicated, and the thing is that we just want to get this working down as you know".

\section{Discussion and Conclusion}

We found that all participants in this study were aware that older adults' privacy will be affected by using ACMDs. Participants noted that decisions about using ACMDs should balance between benefits provided and privacy risks. This is in line with findings from previous studies such as Robinson et al. [15] and Landau et al. [10]. Participants therefore emphasised that any ACMD should strictly limit any collection and sharing of older adults' data. For instance, fall detectors were considered beneficial by all participants as falls are common between elderly people and may cause serious injuries [9]. However, only caregivers who are directly responsible for older adults' wellbeing should receive alerts about falls so they can act to avoid negative consequences.

Furthermore, most of the participants refused to impose using ACMDs on older adults. They emphasised that details on the purpose of the device should be discussed with older adults so they can provide a well-informed consent before using the device. Participants were mostly concerned about privacy as non-invasiveness, non- 
intrusiveness and non-obtrusiveness over information privacy in the use of ACMDs [7]. Only few participants and due to the complexity of privacy policies, were interested in knowing about information privacy including privacy protection measurements (i.e. protections against technical issues such as hacking) applied by service providers. Interestingly, this includes knowing whether the collected data will be shared with third parties and the purposes that the data will be used for before they start using ACMDs.

Caregivers play an important role in influencing older adults' decisions on using monitoring devices [14]. We noticed that caregivers might overlook privacy out of necessity, in particular for those who want to avoid a previous bad experience (e.g. fall or wandering) from happening again in the future. We therefore argue that ACMD developers should consider various older adults' and caregivers' perspectives in relation to privacy such as what data to collect, whom to share it with and when. This requires simplifying privacy notices in terms and conditions by making them short and simple to avoid overwhelming caregivers with unnecessary details. Moreover, developers should consider offering ACMD users different privacy settings so that users are able to tailor their own preferences based on their needs [11].

\section{References}

[1] Alkhatib, S., Waycott, J., \& Buchanan, G. (2019, August). Privacy in Aged Care Monitoring Devices (ACMD): The Developers' Perspective. In Digital Health: Changing the Way Healthcare is Conceptualised and Delivered: Selected Papers from the 27th Australian National Health Informatics Conference (HIC 2019) (Vol. 266, p. 7). IOS Press.

[2] Astell, A. J., McGrath, C., \& Dove, E. (2019). 'That's for old so and so's!': does identity influence older adults' technology adoption decisions?. Ageing \& Society, 1-27.

[3] Caiels, J., Rand, S., Crowther, T., Collins, G., \& Forder, J. (2019). Exploring the views of being a proxy from the perspective of unpaid carers and paid carers: developing a proxy version of the Adult Social Care Outcomes Toolkit (ASCOT). BMC health services research, 19(1), 201.

[4] Caine, K. (2016, May). Local standards for sample size at CHI. In Proceedings of the $2016 \mathrm{CHI}$ conference on human factors in computing systems (pp. 981-992).

[5] Grossi, E., Lucchi, E., Gentile, S., Trabucchi, M., Bellelli, G., \& Morandi, A. (2019). Preliminary investigation of predictors of distress in informal caregivers of patients with delirium superimposed on dementia. Aging clinical and experimental research, 1-6.

[6] Henze, M., Hummen, R., \& Wehrle, K. (2013, May). The cloud needs cross-layer data handling annotations. In 2013 IEEE Security and Privacy Workshops (pp. 18-22). IEEE.

[7] Ienca, M., Wangmo, T., Jotterand, F., Kressig, R. W., \& Elger, B. (2018). Ethical design of intelligent assistive technologies for dementia: a descriptive review. Science and engineering ethics, 24(4), 10351055 .

[8] Krupp, M. M., Rueben, M., Grimm, C. M., \& Smart, W. D. (2017, August). A focus group study of privacy concerns about telepresence robots. In 2017 26th IEEE International Symposium on Robot and Human Interactive Communication (RO-MAN) (pp. 1451-1458). IEEE.

[9] Larizza, M. F., Zukerman, I., Bohnert, F., Busija, L., Bentley, S. A., Russell, R. A., \& Rees, G. (2014). In-home monitoring of older adults with vision impairment: exploring patients', caregivers' and professionals' views. Journal of the American Medical Informatics Association, 21(1), 56-63.

[10] Landau, R., Auslander, G. K., Werner, S., Shoval, N., \& Heinik, J. (2010). Families' and professional caregivers' views of using advanced technology to track people with dementia. Qualitative health research, 20(3), 409-419.

[11] Mahoney, D. F. (2011). An evidence-based adoption of technology model for remote monitoring of elders' daily activities. Ageing international, 36(1), 66-81.

[12] Marshall, B., Cardon, P., Poddar, A., \& Fontenot, R. (2013). Does sample size matter in qualitative research? A review of qualitative interviews in IS research. Journal of Computer Information Systems, 54(1), 11-22.

[13] McCabe, L., \& Innes, A. (2013). Supporting safe walking for people with dementia: User participation in the development of new technology. Gerontechnology, 12(1), 4-15. 
[14] Peek, S. T., Luijkx, K. G., Rijnaard, M. D., Nieboer, M. E., van der Voort, C. S., ... \& Wouters, E. J. (2016). Older adults' reasons for using technology while aging in place. Gerontology, 62(2), 226-237.

[15] Robinson, L., Hutchings, D., Corner, L., Finch, J., Hughes, J., Brittain, K., et al. (2007). Balancing rights and risks: Conflicting perspectives in the management of wandering in dementia. Health, Risk \& Society, 9, 389-406.

[16] Schulz, R., Wahl, H. W., Matthews, J. T., De Vito Dabbs, A., Beach, S. R., \& Czaja, S. J. (2015). Advancing the aging and technology agenda in gerontology. The Gerontologist, 55(5), 724-734.

[17] Superflux. 2015. Uninvited guests. Video. 2015. Retrieved May 15, 2019 from https://vimeo.com/172893044.

[18] Vermeer, Y., Higgs, P., \& Charlesworth, G. (2019). What do we require from surveillance technology? A review of the needs of people with dementia and informal caregivers. Journal of Rehabilitation and Assistive Technologies Engineering, 6, 2055668319869517.

[19] Walsham, G. (2006). Doing interpretive research. European journal of information systems, 15(3), 320330 . 